\title{
Coeficiente de pesimismo relativo ${ }^{1}$
}

\author{
Ángel Samaniego Alcántar* \\ Gerardo Reyes Ruiz** \\ Jordi Bachs Ferrer***
}

\section{Resumen}

El inversor no sólo toma decisiones con base en estados financieros, sino que además toma en cuenta factores externos a la empresa, variables macroeconómicas, expectativas de la economía, entre otros, y refleja este sentir comprando y vendiendo activos. Esta reacción puede ser optimista o pesimista y puede provocar una desviación en la estrategia del inversionista.

Este trabajo pretende ajustar las razones financieras mediante el coeficiente de pesimismo relativo (CPR) que toma en cuenta la relación riesgo-rendimiento. Asimismo, propone una metodología para el cálculo de un indicador relativo de este coeficiente en los inversionistas que participan en un sector del mercado de valores, tomando en cuenta el riesgo asumido por estas empresas en el periodo 2000-2004.

Este indicador ajustó la valoración financiera mediante razones financieras para que converja con la valoración del mercado de valores (desempeño de la acción) con un 84\% de efectividad (veces en que la valoración por razones financieras era igual al desempeño de la acción, entre el total de empresas analizadas). Además, este coeficiente resultó ser un indicador de la tendencia a corto plazo del mercado financiero en los años analizados.

Palabras clave: estudio de eventos, análisis fundamental, criterio de Hurwicz, distancia de Hamming.

Fecha de recepción: 03/12/2007

Fecha de aceptación: 10/03/2008

Clasificación JEL: D81, G14

${ }^{1}$ Los autores agradecen a los árbitros anónimos los comentarios al documento, siendo responsabilidad de los autores cualquier error derivado del mismo.

*Investigador del Instituto Tecnológico y de Estudios Superiores de Occidente.

Correo electrónico: asamanie@iteso.mx.

**Investigador de la Universidad de Barcelona. Correo electrónico: ger_07@hotmail.com

****Profesor-Investigador de la Universidad de Barcelona. Correo electrónico: jbachs@ub.edu

No. 226, septiembre-diciembre 2008:59-72 
Ángel Samaniego Alcántar, Gerardo Reyes Ruiz y Jordi Bachs Ferrer

\title{
Relative pessimism coefficient
}

\begin{abstract}
The investor makes decisions based not only on financial statements, but also taking into account external factors, macroeconomic variables and economy expectations among others. Then he acts upon these views by selling or buying assets. This reaction may be either an optimistic or a pessimistic one, and may cause a variance in the investor's strategy.

This works aims to adjust the financial ratios by means of the relative pessimism coefficient (RPC) which considers the risk-return ratio. It also proposes a methodology for the calculation of a relative indicator of this coefficient in investors who participate in a financial market sector, taking into account the risk assumed by these companies in the 2000-2004 period.

This indicator adjusted the financial valuation through financial ratios so that it would converge with the financial markets valuation (stock performance) with an $84 \%$ effectiveness (times when the valuation through financial ratios equaled the stock performance, divided by total companies analyzed). In addition, this coefficient turned out to be a short-term tendency indicator of the financial market in the years analyzed.
\end{abstract}

JEL codes: D81, G14

Key words: event studies, fundamental analysis, Hurwicz's criterion, Hamming's distance.

\section{Introducción}

T as razones financieras se han utilizado con diferentes fines como, por ejemplo, _seguir la evolución económica financiera de la empresa, medir la eficacia de las diferentes áreas de la empresa, tales como la producción, las ventas y el personal. Las razones financieras han sido un instrumento de mejora en la gestión de la empresa, así como un elemento determinante de la razonabilidad de los estados financieros (Kaufmann y Gil, 1996).

La valoración de los estados financieros mediante razones financieras muchas veces puede indicar mejoras respecto al último reporte de la empresa, sin embargo lo que posiblemente se observa son sólo incrementos (por ejemplo, pasar de un ROE del $16 \%$ a un ROE del 20\%), los cuales tal vez no son lo suficientemente grandes en comparación con un riesgo asumido, que no se está compensando del todo (por ejemplo, el incremento en ROE probablemente fue el mejor de su sector, pero en realidad no compensa la volatilidad del precio de la acción, pudiendo ser la mayor en su sector). Por ello, al realizar la valoración de los estados financieros, éstos necesitan ser ajus- 
tados al riesgo de la empresa o sector. Este trabajo pretende ajustar las razones financieras mediante el coeficiente de pesimismo relativo (CPR), el cual toma en cuenta la relación riesgo-rendimiento y, así, decidir si los incrementos obtenidos en las razones financieras son lo suficientemente grandes.

El análisis fundamental ha sido contrastado como un método para la predicción bursátil porque el mercado descuenta dichos reportes financieros, ya sea favorablemente o negativamente (Abarbanell y Bushee, 1998).

Una manera de ajustar la valoración mediante razones financieras a la relación riesgo-rendimiento es tomando en cuenta las evaluaciones que hace el conjunto de inversionista a la empresa. El inversor no sólo toma decisiones con base en estados financieros, sino que además toma en cuenta factores externos a la empresa: variables macroeconómicas, expectativas de la economía, entre otros, y refleja este sentir comprando y vendiendo activos. Este sentir del conjunto de inversionistas en los momentos en los que sucede el evento (entrega de reportes financieros) trae como consecuencia cambios en el precio de la acción. Esta reacción puede ser optimista o pesimista y este nivel de optimismo o pesimismo ${ }^{2}$ puede provocar una desviación en la estrategia del inversionista (Schipper, 2005) debido a que el inversor incluye otros factores diferentes al análisis de razones financieras para la toma de decisiones.

Lo anterior provoca que no corresponda la valoración financiera con la del mercado de valores (incrementos o decrementos en el precio de la acción). En la literatura se pueden encontrar trabajos donde la reacción del inversionista es subvalorar eventos que afectan directamente a la empresa, algunos de estos trabajos en los últimos años son Kumar y Lee, (2006); Baker y Wurgler, (2006); Brown y Cliff, (2005); Kadiyala y Rau (2004). En resumen, el incremento/decremento de valor se verá en el largo plazo. Esta subvaloración va a ser más alta o más baja dependiendo del nivel de optimismo/pesimismo del conjunto de inversionistas. Para incluir este optimismo/pesimismo en las razones financieras se utiliza el coeficiente de pesimismo relativo, de esta manera se hace coincidir la valoración del mercado con la valoración por razones financieras, esto bajo la hipótesis semifuerte del mercado ${ }^{3}$.

${ }^{2}$ En la literatura anglosajona a este coeficiente se le suele llamar sentiment effect, pero no se encontró alguna metodología que tratará de obtenerlo al relacionar razones financieras y valor accionario de la empresa.

${ }^{3}$ Hipótesis semifuerte del mercado: toda la información publica disponible concerniente a la empresa debe estar reflejada en el precio de acción. 
Este trabajo se encuentra organizado de la siguiente manera: en la sección dos se expone la base de datos utilizada, en la sección tres se describen los métodos utilizados: análisis de componentes principales (ACP), estudio de eventos, criterio de Hurwicz y distancia de Hamming. En la sección cuatro se presentan los resultados obtenidos al contrastar el modelo durante cuatro años y en la sección cinco se analizan los principales resultados, inconvenientes del modelo y las posibles líneas de investigación.

\section{Datos del estudio}

Se utilizaron dos bases de datos:

- $\mathrm{SABI}^{4}$ - Se utilizan las 12 razones financieras del sistema de información, se filtran las empresas para España en el sector de la construcción que se encuentren activas y que coticen en bolsa. De las 28 empresas encontradas se seleccionan aquéllas donde exista información a partir de 1996 a 2004, dando como resultado 11 empresas.

- DataStream - Datos diarios de las cotizaciones desde 1996 a 2004 para las 11 empresas y el índice de bolsa de España IBEX-35.

En la tabla 1 se presentan las razones financieras utilizadas en el estudio.

Tabla 1

Razones financieras utilizadas

\begin{tabular}{|c|c|}
\hline Razón financiera & Cálculo \\
\hline \multicolumn{2}{|l|}{ Actividad } \\
\hline Crecimiento ventas netas $(\%)$ & $=$ porcentaje de cambio en ventas netas \\
\hline Rotación de activos & $=$ importe ventas netas / Total activo \\
\hline Productividad & $\begin{aligned}= & (\text { ventas netas }- \text { costos de ventas }+ \text { otros ingresos y gastos netos }) \\
& / \text { Gastos de personal }\end{aligned}$ \\
\hline $\begin{array}{r}\text { Crecimiento Valor } \\
\text { Añadido (\%) }\end{array}$ & $\begin{array}{l}\text { = porcentaje de cambio en valor añadido } \\
\text { Valor añadido }=\text { ventas netas }+ \text { otros ingresos por ventas }- \text { gastos de } \\
\text { ventas }- \text { otros gastos de ventas }\end{array}$ \\
\hline \multicolumn{2}{|l|}{ Rentabilidad } \\
\hline $\begin{array}{r}\text { Rentabilidad económica (\%) } \\
\text { Rentabilidad financiera (\%) }\end{array}$ & $\begin{array}{l}=\text { utilidad del ejercicio / total activo } \\
=\text { utilidad del ejercicio / fondos propios }\end{array}$ \\
\hline Gastos financieros $(\%)$ & $=$ costo integral de financiamiento $/$ ventas netas \\
\hline \multicolumn{2}{|l|}{ Solvencia } \\
\hline Endeudamiento (\%) & $=($ total pasivo - capital contable $) /$ total pasivo \\
\hline $\begin{array}{r}\text { Capital contable / Capital } \\
\text { invertido (\%) }\end{array}$ & $=$ capital contable $/$ (capital contable + pasivo a largo plazo $)$ \\
\hline
\end{tabular}

${ }^{4}$ Sistema de Análisis de Balances Ibéricos. 
Coeficiente de pesimismo relativo

\begin{tabular}{|c|c|}
\hline Capacidad devolución & $\begin{aligned}= & \text { (acreedores a LP }+ \text { pasivo circulante }) / \text { (importe neto en ventas }+ \\
& \text { depreciaciones y amortizaciones de activos }+ \text { pérdida de créditos } \\
& \text { incobrables }+ \text { variaciones provisiones de inversiones financieras })\end{aligned}$ \\
\hline \multicolumn{2}{|l|}{ Liquidez } \\
\hline Liquidez general & $=$ activo circulante $/$ pasivo circulante \\
\hline Liquidez inmediata & $=$ efectivo e inversiones temporales / pasivo circulante \\
\hline
\end{tabular}

Fuente: SABI, se filtró la base de datos por sector de la construcción, empresas activas y que coticen en bolsa. Se obtuvo un total de 28 empresas, de las cuales se seleccionaron 11 por tener información de 1996 a 2004

\section{Metodología}

Primero se introduce al criterio de decisión de Hurwicz, posteriormente se analizan los pasos por seguir en la metodología propuesta.

La característica principal de los problemas de decisión en ambiente de incertidumbre reside en el grado de conocimiento que el decisor tiene del entorno. En algunos casos el decisor conoce su sentir pero no tiene otra información, por eso no es capaz de asignarle un grado de probabilidad a dicho evento. El criterio de decisión de Hurwicz (Hurwick, 1951 y García et al., 2002) se refiere a que el decisor es capaz de medir su grado de pesimismo relativo a través de un coeficiente $C P R \in[0,1]$.

$$
A_{i j} \rightarrow c_{i j}=C P R_{j} \cdot m_{i j}+\left(1-C P R_{j}\right) \cdot M_{i j}
$$

donde:
$A_{i}$ es la alternativa a valorar (razón financiera en este estudio)
$c_{i}$ es el valor de la alternativa
$m_{i}$ es la peor valoración
$M_{i}$ es la mejor valoración
$i$ es la empresa
$j \quad$ es el año de referencia

El valor que el decisor le da a una alternativa estará relacionada por su nivel de pesimismo u optimismo $(C P R)$ que tenga sobre la alternativa $i$, en el año $j$.

El criterio de decisión de Hurwicz se utilizará primero para valorar cada una de las cuatro razones financieras en cada año; después se valora el conjunto de las cuatro razones financieras, la cual se realiza mediante razones financieras (ver pasos 1 al 4). 
En la tabla 2 se explican los pasos por seguir para aplicar la metodología.

Tabla 2

Pasos por seguir de la metodología propuesta

\begin{tabular}{|ll|}
\hline Paso 1: & Selección de razones financieras (RF) por utilizar. \\
Paso 2: & Creación del intervalo donde se moverá la RF, con base al CPR. \\
Paso 3: & Valoración de la empresa por cada RF. \\
Paso 4: & Valoración de la empresa por RF (incluyendo todas las RF). \\
Paso 5: & Valoración de la empresa por los mercados financieros (rendimiento anormal). \\
Paso 6: & Relacionar la valoración por RF con la valoración por mercados financieros. \\
Paso 7: & Valoración del modelo (porcentaje de veces que coinciden ambos modelos). \\
Paso 8: & Búsqueda del CPR óptimo donde la valoración del modelo es el máximo. \\
\hline \hline
\end{tabular}

Nota: $R F$ - razón (es) financiera (s)

Tabla 3

Razones financieras seleccionadas por ACP

Cada columna indica las $R F$ con mayor representatividad tienen en la varianza y menor correlación entre ellos para cada año.

\begin{tabular}{|c|c|c|c|c|c|}
\hline Razón financiera & 2000 & 2001 & 2002 & 2003 & 2004 \\
\hline \multicolumn{6}{|l|}{ Actividad } \\
\hline Crecimiento ventas netas $(\%)$ & & $\mathrm{x}$ & & & \\
\hline Rotación de activos & & $\mathrm{x}$ & & & \\
\hline \multicolumn{6}{|l|}{ Productividad } \\
\hline Crecimiento Valor Añadido (\%) & & & & $x$ & $x$ \\
\hline \multicolumn{6}{|l|}{ Rentabilidad } \\
\hline Rentabilidad económica (\%) & & $\mathrm{x}$ & & $\mathrm{x}$ & $\mathrm{x}$ \\
\hline Rentabilidad financiera (\%) & & & $x$ & & \\
\hline Gastos financieros (\%) & $x$ & & $x$ & $x$ & \\
\hline \multicolumn{6}{|l|}{ Solvencia } \\
\hline Endeudamiento $(\%)$ & $\mathrm{x}$ & $\mathrm{x}$ & & & \\
\hline Capital contable / Capital invertido (\%) & & & & $x$ & \\
\hline Capacidad devolución & & & & & $\mathrm{x}$ \\
\hline \multicolumn{6}{|l|}{ Liquidez } \\
\hline Liquidez general & $\mathrm{x}$ & & $\mathrm{x}$ & & \\
\hline Liquidez inmediata & $\mathrm{x}$ & & & & $\mathrm{x}$ \\
\hline Varianza total explicada & 88.91 & 88.43 & 83.26 & 88.49 & 85.47 \\
\hline
\end{tabular}

Nota: En algunos casos se utilizaron los componentes rotados mediante Varimax 
Paso 1: Se busca que razones financieras tienen mayor representatividad de la varianza en la empresa. Para los reportes financieros comprendidos en el periodo 20002004 se llevó a cabo un ACP (ver Uriel y Aldás, 2005) con la finalidad de detectar las $R F$ que mayor representatividad tienen en la varianza y menor correlación tengan entre ellas para cada año donde se realizó la contrastación del modelo (ver tabla 3). Los resultados en la tabla 3 muestran como cada año se van seleccionando razones financieras diferentes, esto es congruente debido a que cada año no es el mismo, habrá años donde existe mayor volatilidad en una razón financiera que en otra. Si no existe un cambio en una razón financiera de un año a otro no sería congruente seleccionarla.

Tabla 4

Porcentaje de varianza explicada de cada componente en cada año

\begin{tabular}{|rccccc|}
\hline Componente principal & 2000 & 2001 & 2002 & 2003 & 2004 \\
\hline 1ra componente & 41.20 & 35.97 & 45.13 & 32.96 & 35.29 \\
2da componente & 22.32 & 21.31 & 27.89 & 27.21 & 25.08 \\
3ra componente & 15.09 & 18.09 & 10.24 & 16.13 & 14.11 \\
4ta componente & 10.29 & 13.06 & & 12.18 & 10.99 \\
& & & & & \\
Varianza explicada & 88.91 & 88.43 & 83.26 & 88.49 & 85.47 \\
\hline \hline
\end{tabular}

Paso 2: En este paso se incorpora la incertidumbre al modelo. Para el conjunto de $R F$ se construyó un intervalo de incertidumbre de la siguiente manera:

$$
\left[R F_{i}-2 \sigma_{i} C P R \quad, \quad R F_{i}+2 \sigma_{i}(1-C P R)\right]
$$

con $C P R \in[0,1], i$ es la razón financiera $\{1,2,3,4\}$ para cada año.

Se tomó dos veces la desviación estándar de la $R F_{i}$ porque fue el valor que le dio mayor sensibilidad al modelo; con base en lo anterior, es el intervalo donde el inversor piensa que se puede mover $R F_{i}$ para cada empresa. Este intervalo discrimina una empresa con un alto riesgo de aquella que no lo tiene; es decir:

$$
C P R[\Phi(n, R F)]
$$

donde:

$\Phi$ es el comportamiento del mercado de valores en un determinado periodo de tiempo $n$.

$\mathrm{CPR}$ es el coeficiente de pesimismo relativo para ese periodo. 
Es fácil apreciar con base en la ecuación (2) que para $C P R=1$ en la ecuación (4) se está en un entorno pesimista mientras que para $C P R=0$ en la ecuación (5) se está en un ambiente optimista.

$$
\begin{aligned}
& {[R F-2 \sigma, R F]} \\
& {[R F, R F+2 \sigma]}
\end{aligned}
$$

Se escribe este intervalo de la forma:

$$
\begin{gathered}
{\left[a_{1}, a_{2}\right]} \\
\operatorname{con} C P R \in[0,1]
\end{gathered}
$$

Paso 3: Una vez calculado el intervalo de incertidumbre para cada $R F$ seleccionada en relación con su riesgo ${ }^{5}(\sigma)$ asumido se calcula su correspondiente distancia de Hamming (Kaufmann, Gil, 1996). Esta distancia es la suma en valor absoluto de cada intervalo de la $R F_{i}$ con su máximo (de todas las empresas comparadas en el año correspondiente). Entre menor diferencia exista en ambos intervalos menor será la distancia con el mejor resultado posible, por lo que se diría que el valor $R F_{i}$ fue el mejor entre los posibles. En ocasiones se buscará no la menor distancia con el máximo posible, sino la máxima distancia debido a que es un $R F$ que se desea sea la menos (por ejemplo, nivel de apalancamiento).

La correspondiente distancia de Hamming igualando (2) a (6):

$$
d\left(R F_{i}\right)=\left|a_{1_{i}}-\bigvee_{i=1}^{n} a_{1_{i}}\right|+\left|a_{2_{i}}-\bigvee_{i=1}^{n} a_{2_{i}}\right|
$$

donde:

$$
\bigvee_{i=1}^{n} \text { denota el máximo }
$$

Esto nos dará una distancia para cada $R F$ en cada empresa y para cada año.

Se ordenan las distancias en cada año y se obtiene un ranking por empresa para cada grupo de $R F$.

${ }^{5}$ Se tomaron cuatro años para el calculo de la desviación estándar. 
Coeficiente de pesimismo relativo

Paso 4: Del paso anterior no se tiene un ranking donde se incluyan las $4 R F$ en cada año, aquí es donde se toman en cuenta y el resultado será la valoración por $R F$. Se calcula un ranking para cada año utilizando como ponderación el porcentaje de varianza que explica cada componente principal (ver tabla 4).

$$
\operatorname{ranking}_{i}=\sum w_{i} \cdot \operatorname{ranking}\left(R F_{\mathrm{i}}\right)
$$

Paso 5: Para la valoración de los mercados financieros se realiza un estudio de eventos (ver Bodie et al., 2002 y Campbell et al., 1997). donde el evento es el reporte financiero. Se calcula el rendimiento esperado $\left(\mathrm{E}\left[R_{t}\right]\right)$ por el inversor, esto mediante una regresión dinámica ${ }^{6}$ para cada año durante los próximos tres meses después de fin de año (asumiendo que en esos tres meses la empresa entregará su reporte a bolsa).

Para ello se tomaron cuatro años del rendimiento diario de la acción en bolsa $(R)$ y el rendimiento diario del IBEX-35 $(R M)$. El método de estimación fue por el modelo de mercado (market model no se debe confundir con el CAPM):

$$
E\left[R_{t}\right]=a_{t}+R M_{t} \beta_{t}+e_{t}
$$

donde:

$a$ es la intersección del resultado mediante OLS (Ordinary Least Square).

$\beta$ es la sensibilidad de la acción de la empresa con el mercado (índice de bolsa, IBEX-35).

$R M$ es el rendimiento del índice de bolsa, IBEX-35.

$e$ es el termino de error.

$t$ son los días donde ocurre el evento (durante tres meses después del cierre del año).

Y donde su correspondiente rendimiento anormal acumulado (Cumulative Abnormal Return, CAR) para los tres meses en cada año:

$$
C A R_{t}=\sum\left(R_{t}-E\left[R_{t}\right]\right)
$$

${ }^{6} \mathrm{El}$ rango de datos son cuatro años con relación al rendimiento de la empresa (variable dependiente) y el IBEX-35 (variable independiente). Se fue moviendo el rango de datos para el rendimiento esperado del siguiente día, esto fue durante tres meses. 
donde:

$R_{t}$ es el rendimiento de la acción de la empresa $i$ en el periodo $t$.

Este resultado nos dirá si es positivo que el mercado reaccione positivamente a los reportes financieros y a los sentimientos del inversor (otros factores que el inversor toma en cuenta) en el momento de tomar decisiones; y si es negativo lo contrario a lo comentado.

Figura 1

Rendimiento anormal acumulado: Acciona 2001-2004

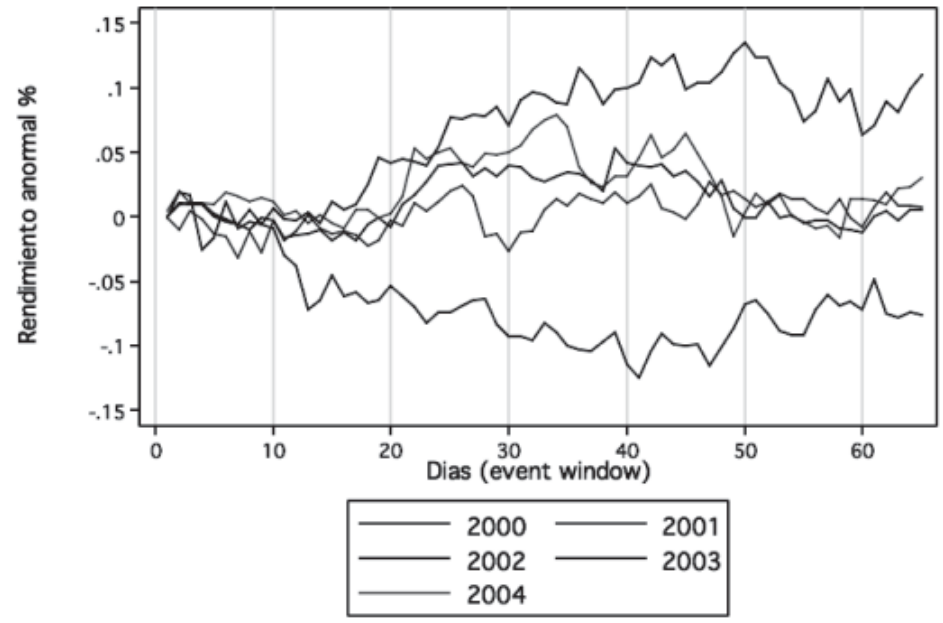

El CAR de la empresa Acciona durante los primeros tres meses en los cuatro años analizados (2000-2004). La columna vertical indica rendimiento, por ejemplo, el rendimiento acumulado para el año 2000 es negativo, indicando una reacción negativa al evento, reporte financiero de fin de año.

Ahora bien, si el $C A R$ es positivo $(C A R=1)$ significa que el evento provocó una diferencia positiva entre el rendimiento de la empresa y el rendimiento esperado (en el periodo de estudio). Esto tomando en cuenta el riesgo asumido. Si el CAR es negativo $(C A R=0)$ entonces el evento incluyó de forma negativa lo que sucede en la figura 1 para el año 2000. Este resultado será la valoración por mercados financieros.

Paso 6: El objetivo es relacionar la valoración por $R F$ con la valoración por mercados financieros, para lo cual se utiliza una variable dicotómica (acierto $=1$, fallo $=0$ ), la cual se construyó utilizando la siguiente discriminación.

Para una empresa con un $C A R=1$ (valoración positiva para los mercados financieros), su valoración por $R F$ o número de ranking obtenido debe ser menor o igual al total de 
Coeficiente de pesimismo relativo

frecuencias del $C A R$ (suma de los $C A R=1$ ) en el año analizado. En otras palabras, si una empresa está en quinto lugar en el ranking por $R F$ y tuvo un valoración positiva por los mercados financieros $(C A R=1)$, teniendo ese año sólo cuatro empresas con un $C A R=1$; no corresponden ambas valoraciones debido a que un quinto lugar no corresponde con sólo cuatro empresas clasificas como positivas por los mercados financieros. En el caso anterior se clasifica como fallido (fallo $=0$ ).

Paso 7: Se calcula la probabilidad de aciertos, es el resultado del cociente entre el total de aciertos y el numero de empresas, para cada en el año analizado (utilizando los datos del paso 6).

Paso 8: Se identifica el óptimo $\alpha$, donde se encuentra la máxima probabilidad de aciertos (ver tabla 5).

Tabla 5

Probabilidad de aciertos para cada coeficiente de pesimismo relativo

\begin{tabular}{|crrrrr|}
\hline \multirow{2}{*}{ Año } & \multicolumn{5}{c|}{ Coeficiente de pesimismo relativo } \\
\cline { 2 - 6 } 2000 & 0 & 0.25 & 0.5 & 0.75 & 1 \\
2001 & $45 \%$ & $64 \%$ & $\mathbf{8 2 \%}$ & $64 \%$ & $64 \%$ \\
2002 & $73 \%$ & $64 \%$ & $73 \%$ & $\mathbf{8 2} \%$ & $82 \%$ \\
2003 & $64 \%$ & $73 \%$ & $73 \%$ & $\mathbf{8 2} \%$ & $73 \%$ \\
2004 & $\mathbf{8 3 \%}$ & $75 \%$ & $75 \%$ & $75 \%$ & $75 \%$ \\
\hline \hline
\end{tabular}

Se realizaron varias iteraciones del modelo con diferente $C P R$, el cual daría diferentes niveles de efectividad, por ejemplo, para el 2000 el coeficiente de 0.5 da la mayor probabilidad de aciertos para ese año de $82 \%$.

\section{Resultados empíricos}

En la tabla 5 se encuentran los $C P R$ utilizados para cada año donde la intersección entre el año y el correspondiente $C P R$ será la probabilidad de aciertos, la cual viene dada por el total de aciertos del modelo entre el número de empresas. Se pueden observar algunas inconsistencias en los resultados.

El CPR para 2001 y 2003, no es lo suficientemente sensible para diferenciar la efectividad del modelo en un $82 \%$ y $75 \%$, respectivamente.

Para 2002 se pueden apreciar oscilaciones en el nivel de efectividad, indicándonos que hay otra posible variable de influencia en el modelo. 
Los rankings calculados con este CPR óptimo para el modelo algunas de las veces no corresponden con el nivel del CAR. En otras palabras, a un CAR alto no necesariamente significa que la empresa está en los primeros lugares en el ranking.

En el periodo analizado se obtuvo un promedio del $84 \%$ de aciertos en el modelo (ver tabla 6).

Tabla 6

Probabilidad de aciertos del modelo utilizando CPR óptimos

\begin{tabular}{ccc}
\hline Año & CPR & Efectividad \\
\hline 2000 & 0.5 & $81.8 \%$ \\
2001 & 0.75 & $81.8 \%$ \\
2002 & 0.75 & $81.8 \%$ \\
2003 & 0 & $83.3 \%$ \\
2004 & 0.5 & $91.7 \%$ \\
& Promedio & $\mathbf{8 4 . 1 \%}$ \\
\hline
\end{tabular}

Tomando en cuenta los CPR mayores de cada año, de la tabla 4, se obtiene el promedio de los 4 años, que se resume en la siguiente tabla.

Como se muestra en la figura 2, el CPR calculado para el periodo 2000-2004 resultó consistente con el comportamiento del IBEX-35 debido a que una caída en el índice prolongada puede ir acompaña de altos niveles de pesimismo (75\%) bajo la hipótesis de una variación baja entre los niveles de pesimismo de los sectores económicos.

Figura 2

IBEX-35:2000-2004

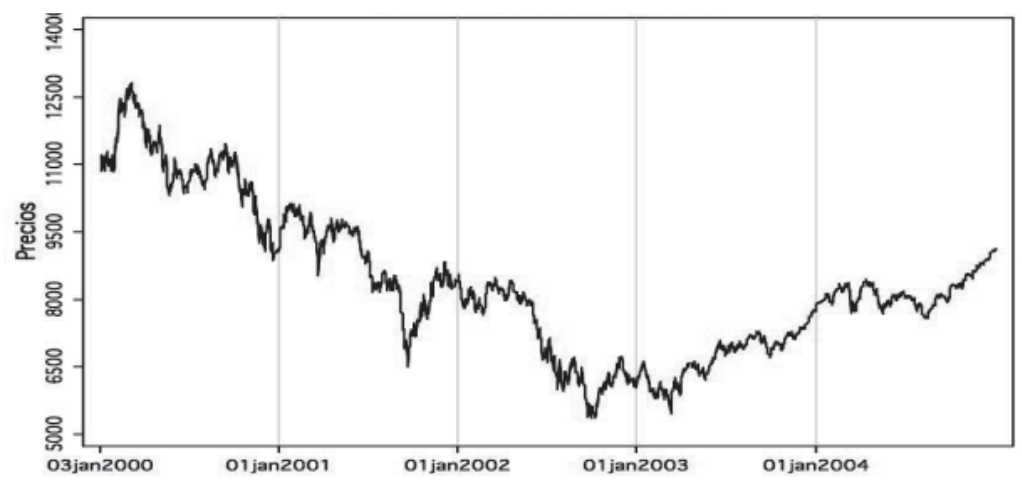

Durante 2001 a 2002 el $\alpha=0.75$, indicando el mayor pesimismo entre 2000-2004, por lo que el índice IBEX-35 presenta su mayor caída. En 2000 y 2004 un $\alpha=0.5$ indica un pesimismo menor donde se comprueba con caídas y alzas en el índice IBEX-35. En 2003 un $\alpha=0$ donde la reacción del índice es positiva. 
Coeficiente de pesimismo relativo

\section{Conclusiones}

La metodología propuesta ajustó los niveles de las razones financieras de la emisora durante el último cuarto de cada año (entre 2000-2004), utilizando el CPR del inversor para el sector de la construcción en España. De esta manera las razones financieras ajustadas por el coeficiente de pesimismo son consistentes con el desempeño (rendimiento y riesgo) de la emisora en los mercados financieros; por ejemplo, algunas empresas que tenían un buen desempeño en sus razones financieras antes de aplicarles la metodología, después indicaban un mal desempeño debido a que el rendimiento de la emisora no fue lo suficientemente alto en relación con el rendimiento que se recibe a un riesgo dado (emisoras con mayor riesgo se les exige mayor rendimiento).

Este coeficiente de pesimismo ajustó las razones financieras con base al rendimiento y riesgo de la emisora con un $84 \%$ de aciertos (promedio de casos favorables entre total de casos). Se puede pensar que el $16 \%$ restante se debe al pesimismo específico de la emisora siendo el $84 \%$ al sector analizado.

Se encontró que el coeficiente de pesimismo era congruente con el rendimiento del IBEX-35, entre 2000-2004. Se observó que a mayor pesimismo mayor tendencia a la baja del índice.

La metodología asume varios supuestos: hipótesis semi-fuerte del mercado, las hipótesis de OLS, entre otros, los cuales podrían ser de fácil falseabilidad.

Por lo que puede ser criticable en muchos sentidos, la idea del modelo es percibir la realidad como problemas híbridos en el sentido que la información puede ser cierta (conocemos la distribución de probabilidad) e incierta (desconocemos la distribución de probabilidad) donde el coeficiente de pesimismo ajusta la información incierta.

Por los puntos anteriores este coeficiente de pesimismo relativo tiene que ser contrastado para otros periodos y mercados.

Este trabajo puede derivar las siguientes líneas de investigación:

- Que tan consistente puede ser para detectar momentos para la colocación de capital.

- La utilización de otros modelos para el calculo del CAR $\left(\mathrm{CCAPM}^{7}, \mathrm{FF}\right.$ model $^{8}$, redes neuronales).

- Contrastar la metodología para otros sectores o mercados financieros.

${ }^{7}$ Conditional CAPM, ver Cochrane, 2001.

${ }^{8}$ Fama and French model, ver Cochrane, 2001. 


\section{Referencias}

Abarbanell, J. S. y J. Bushee (1998), "Abnormal returns to a fundamental analysis strategy", The Accounting Review vol. 73, pp. 19-45.

BAKER, M. y J. Wurgler (2006), "Investor sentiment and the cross-section of stock returns”, Journal of Finance, vol. 61, pp. 1645-1680

Bodie, Z., A. Kane y A. Marcus (2002), Investments, 5a, ed., McGraw-Hill.

Brown, G. W. y M. T. CLIFF (2005), "Investor sentiment and asset valuation", Journal of Business, vol. 78, pp. 405-440

Campbell, J., W. Lo y A. MacKinlay (1997), The econometrics of financial markets, Princeton University Press, New Jersey.

Cochrane, J. (2001), Asset pricing, Princeton University Press, New Jersey.

García, J., E. Martínez, R. Redondo y C. Del Campo (2002), Métodos de decisión: Ejercicios Resueltos, Pearson Educación, Madrid.

Hurwicz, L. (1951), "Optimality criteria for decision making under ignorance”, Cowles Commission Discussion Paper, No. 370.

Kadiyala, P. y P. R. RaU (2004). "Investor reaction to corporate event announcements: Underreaction or overreaction?”, Journal of Business, vol. 77, pp. 357-386

Kaufmann, A. y J. GiL (1996), Introducción de la teoría de subconjuntos borrosos a la gestión de las empresas, Milladoiro.

Kumar, A. y C. M. C. Lee (2006), "Retail investor sentiment and return comovements", Journal of Finance, vol. 61, pp. 2451-2486

Schipper, B. (2005), "The Evolutionary Stability of Optimism, Pessimism and Complete Ignorante", GESY, Discussion Paper, vol. 68, pp. 1-31.

Uriel E. y J. Aldás (2005), Análisis Multivariante Aplicado, Thomson, Madrid. 